\title{
Le scénario comme outil pédagogique
}

Pourquoi, comment et pour qui ?

\section{Shaeda Isani}

\section{(2) OpenEdition}

\section{Journals}

Édition électronique

URL : http://journals.openedition.org/asp/3643

DOI : $10.4000 /$ asp.3643

ISSN : 2108-6354

Éditeur

Groupe d'étude et de recherche en anglais de spécialité

\section{Édition imprimée}

Date de publication : 1 décembre 1996

Pagination : 313-326

ISSN : 1246-8185

\section{Référence électronique}

Shaeda Isani, «Le scénario comme outil pédagogique », ASp [En ligne], 11-14 | 1996, mis en ligne le 12 juillet 2013, consulté le 20 avril 2019. URL : http://journals.openedition.org/asp/3643 ; DOI : 10.4000/ asp.3643

Ce document a été généré automatiquement le 20 avril 2019

Tous droits réservés 


\section{Le scénario comme outil pédagogique}

Pourquoi, comment et pour qui ?

\section{Shaeda Isani}

1 Introduction

\section{Pourquoi?}

Backwash ou le lien de causalité entre scénario d'évaluation et scénario pédagogique

En guise d'introduction, j'aimerais évoquer un important travail de recherche et de réflexion commencé depuis 1993 dans le cadre de la création du Diplôme de compétence en langue (DCL), un système d'évaluation qui mesure la compétence en langue à travers un outil d'évaluation relativement novateur, le scénario d'évaluation. Une des principales préoccupations des travaux qui ont conduit à la conception et à l'élaboration des outils docimologiques fut celle de veiller au phénomène du backwash (Prodromou 1995) c'est-àdire, aux retombées qu'un système d'évaluation produit sur les enseignements qui lui sont inféodés. C'est ce phénomène de backwash qui constitue l'important lien naturel qui devrait exister entre le choix des objectifs pédagogiques d'une part, et le type d'évaluation qui les sanctionne, d'autre part. À ce titre, il représente un des facteurs les plus motivants de la mise en œuvre d'un système d'évaluation car il peut, à lui tout seul, opérer des changements très puissants - en positif ou en négatif - au niveau de l'apprentissage et de l'enseignement des langues.

Il existe deux principaux types de backwash : «beneficial (or positive) backwash » lorsque les retombées engendrent des effets jugés positifs, et "harmful (ou negative) backwash» lorsqu'un examen présente des caractéristiques qui se répercutent de façon jugée négative sur les enseignements qui s'y rapportent. Un exemple souvent cité pour illustrer le phénomène de "negative backwash » concerne les examens construits autour des QCM de grammaire. La logique du backwash entre impitoyablement en fonction et nous assistons au spectacle déplorable d'étudiants-candidats passant des heures et des heures sur des aspects somme toute secondaires, tels que l'apprentissage théorique du code 
linguistique et la grammaire de l'exception d'une part, l'acquisition des trucs et des astuces censés faciliter la réussite aux examens d'autre part. Soucieux d'éviter des effets aussi pervers, les promoteurs du DCL ont eu pour préoccupation majeure de déclencher un phénomène de "positive backwash" autrement dit, en ce qui nous concerne, nous formateurs, d'orienter les formations s'y rapportant vers une authentique pratique de la langue, ce qui explique le choix du scénario comme support de l'évaluation.

\subsection{Le scénario et la notion de tâches communicative transversales}

Comment le scénario d'évaluation peut-il remplir ce contrat ${ }^{1}$ ? Cette question soulève toute la problématique des aspects à privilégier - et donc $a$ fortiori, à mettre de côté lors de l'élaboration des programmes d'enseignement-apprentissage des langues étrangères et leur évaluation. Ainsi, l'équipe de création du DCL a eu à se pencher sur l'épineuse tâche de "réduire » la langue à des proportions gérables sans violer l'aspect holistique qui la caractérise. La réponse a été trouvée grâce à une enquête importante conduite pour recenser les besoins langagiers du public visé (public «post-bac» et professionnel) et l'analyse des tâches communicatives les plus récurrentes. Ainsi, ont pu être recensées certaines tâches communicatives qui sont transversales à la grande majorité des pratiques socio-professionnelles qui caractérisent le monde professionnel. Le scénario s'est ensuite imposé comme le support idéal de la mise en forme de ces tâches communicatives transversales, non seulement dans le domaine de l'évaluation mais également dans celui de l'enseignement.

\subsection{Qu'est-ce qu'un scénario?}

5 La notion de tâche communicative transversale nous permet de définir, ici, le scénario comme un enchaînement de tâches communicatives thématiquement liées qui conduit à la prise d'une décision. Les tâches communicatives qui composent le scénario s'articulent autour de la quête, de la sélection et de la restitution de l'information, activités que nous sommes tous appelés à accomplir quotidiennement en langue maternelle.

Le scénario s'articule autour d'un enchaînement de cinq macro-tâches communicatives transversales (cf. figure 1). 
Figure 1. Le scénario et l'enchaînement des tâches communicatives transversales

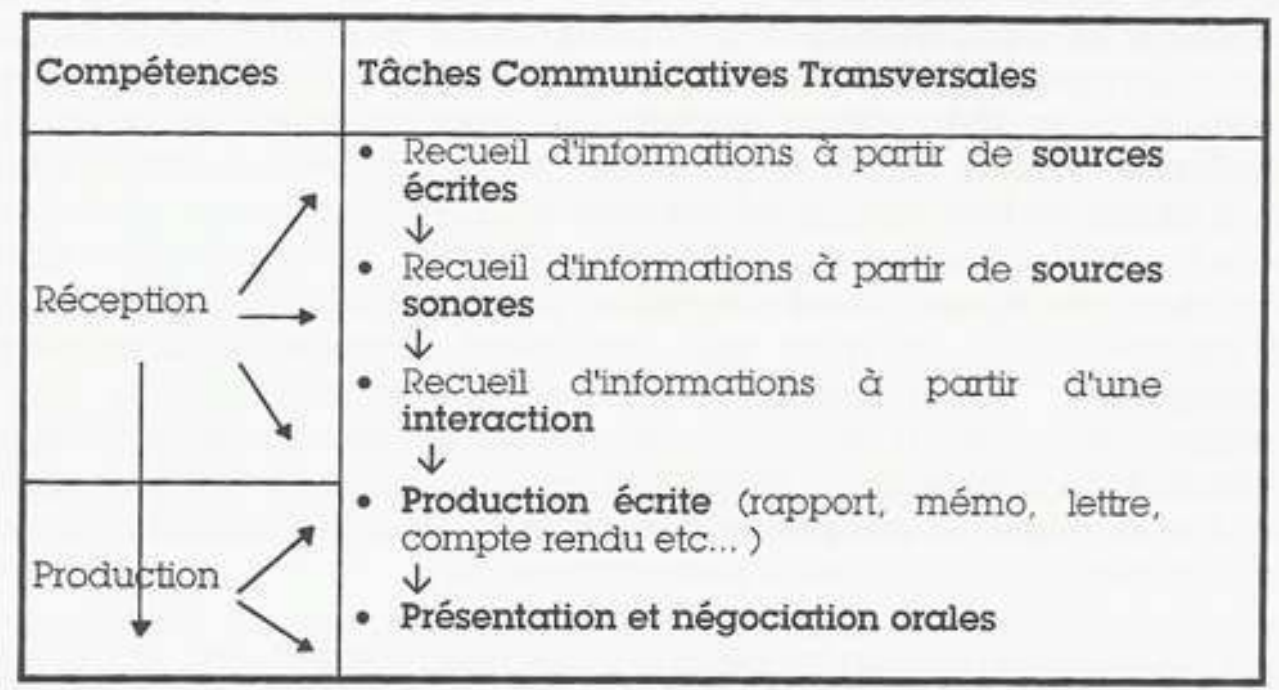

7 Il est important de souligner à ce stade que le concept du scénario ne s'arrête pas à un simple recueil d'informations, passif et gratuit. Il repose sur la dynamique de l'appropriation et de la mobilisation des informations recueillies en vue d'une prise de décision personnalisée qui est ensuite communiquée à travers les deux tâches de production qui suivent les phases de réception. Ainsi, nous disposons d'un outil performant qui satisfait pleinement aux exigences d'un enseignement-apprentissage visant l'acquisition d'une compétence de communication, à savoir, un apprentissage actif, centré sur l'apprenant et sur une pratique de la langue rapprochée de la vie réelle.

\section{Comment?}

\section{Fabrication et exploitation du scénario en tant qu'outil pédagogique}

Malgré le fait qu'un des atouts majeurs du scénario en tant qu'outil pédagogique est qu'il rapproche les objectifs des enseignements qui l'exploitent d'une pratique de la langue plus authentique, il reste un outil pédagogique peu exploité dans le domaine de l'enseignement des langues étrangères en France ${ }^{2}$. Par conséquent, il ne serait pas étonnant que des enseignants soient quelque peu intimidés par l'idée de fabriquer euxmêmes un outil encore peu expérimenté dans leur environnement immédiat. L'expérience montre cependant qu'il ne s'agit nullement d'un travail qui se situe au-delà des capacités de l'enseignant moyen: je me propose maintenant d'examiner les différentes composantes du scénario pédagogique, et la manière de les élaborer.

9 La construction d'un scénario pédagogique peut se décomposer en quatre étapes chronologiques :

- choix du thème et collecte des documents,

- définition de la double problématique et rédaction de la mise en situation,

- construction des phases du scénario,

- fabrication des grilles de guidage. 


\subsection{Choix du thème et collecte de documents}

10 Les cinq tâches communicatives transversales retenues pour le scénario correspondent aux besoins et à la pratique de la langue de la majorité du public concerné et constituent ainsi le pôle fixe du scénario. En revanche, le choix de la thématique permet de "personnaliser » les besoins et les intérêts de chaque public et constitue par conséquent la pierre de touche de la très grande adaptabilité du scénario.

11 Si le choix du thème est déterminé en fonction des besoins et des intérêts de l'apprenant, il est aussi très largement tributaire des ressources de l'enseignant. Bien évidemment, si l'enseignant exerce dans un établissement nanti d'un centre de ressources bien garni, le problème est considérablement réduit. Malheureusement, dans la vaste majorité des cas, l'enseignant est son propre centre de ressources et doit recourir au bon vieux système D pour obtenir des documents.

12 Si la collecte de documents écrits pose relativement peu de problèmes, celle des documents sonores peut demander plus d'initiative. Mais encore une fois, elle ne relève pas de l'insurmontable. Tous les enseignants des langues majoritairement enseignées disposent d'au moins trois sources facilement accessibles qui sont de véritables mines d'or en matière de documents exploitables dans la fabrication de la phase de réception orale du scénario :

- informations télévisées en Vo (CBS news en anglais, Euronews en espagnol, italien, et allemand, par exemple),

- des collègues et/ou des amis (natifs, non-natifs) qui veulent bien faire un enregistrement,

- films vidéo en Vo.

13 Par conséquent, une fois dans la dynamique de la fabrication des scénarii, la collecte des documents à la fois écrits et sonores devient une véritable manie et le problème de l'enseignant se situe davantage au niveau de la gestion de la pléthore que de la pénurie.

\subsection{Définition de la double problématique et rédaction de la mise en situation}

Comme nous l'avons dit précédemment, le scénario n'est pas un simple exercice de lecture et de compréhension de documents écrits et sonores mais repose sur la dynamisation des informations recueillies. Cette dynamisation est ancrée dans une double problématique dont la résolution exige non seulement un certain nombre d'informations mais également une réflexion et prise de position personnelle. La problématique est double car dans le cadre d'une problématique générale vient s'inscrire une problématique individuelle. Ainsi, si l'on prend un exemple dans le domaine du FLE, par exemple, l'enseignant pourra proposer des informations sur une grève des transports en France qui seront les éléments constitutifs de la définition de la problématique générale. Sur cette problématique générale, se greffe la problématique individuelle : ex. je suis étudiant et il y a six mois, ma grand-mère m'a offert des billets pour le concert des Pink Floyd. Avec la grève des transports, comment pourrais-je faire pour aller d'Aix à Paris pour assister à ce concert?

La définition de la double problématique est soumise à l'apprenant sous forme d'une mise en situation. Lorsqu'il s'agit d'un contexte d'évaluation visant un public homogène 
du point de vue de la langue source, la mise en situation est, par principe, rédigée dans cette langue de sorte à éviter toute confusion sur le plan de la compréhension des consignes. En situation didactique, par contre, la mise en situation peut constituer un élément supplémentaire de travail en langue cible et, à ce titre, être rédigée dans cette langue. Quelle que soit la langue retenue, dans l'intérêt du bon déroulement du scénario, il importe d'être très vigilant sur la clarté des paramètres définissant la problématique générale et les consignes régissant le rôle que l'apprenant aurait à jouer au niveau de la problématique individuelle.

\subsection{Fabrication des phases du scénario}

Chaque phase de réception doit, dans la mesure du possible, contenir des informations qui lui sont exclusives, sans recoupements inutiles.

Si les deux phases de compréhension, l'écrit et l'oral, posent relativement peu de problèmes, la phase d'interaction peut, de prime abord, paraître contraignante à organiser, en raison de la nécessité d'avoir un interlocuteur, détenteur d'informations. Mais, on se rend vite compte que dans le contexte du scénario pédagogique, il existe plusieurs façons d'organiser cette phase, qui, rappelons-le, constitue la composante la plus originale et motivante du scénario. Voici quatre possibilités.

\section{L'enseignant}

Les apprenants viennent recueillir les informations auprès de l'enseignant lui-même. Cette configuration peut présenter quelques difficultés dans le cas de classes nombreuses, bien que le recours aux lecteurs soit souvent envisageable.

Les paires

19 Ancrée dans l'approche fondée sur pair learning, il s'agit d'une activité qui exploite les paires comme co-constructeurs de savoir. La classe est divisée tout simplement en deux, trois ou quatre groupes possédant des informations différentes. La tâche à accomplir consiste à recueillir les renseignements manquants auprès des paires qui les possèdent.

\section{Le réseau " téléphonique "}

20 Certains laboratoires de langue permettent aux apprenants de communiquer entre eux en réseau téléphonique. Ainsi, certains apprenants possèdent des informations dont d'autres ont besoin, et vice versa. Les apprenants s'appellent jusqu'à ce qu'ils aient obtenu toutes les informations dont ils ont besoin.

\section{Des sources extérieures à l'établissement}

21 Selon le type de scénario et le contexte d'enseignement, la phase d'interaction peut être envisagée sous forme d'activité extérieure à la classe, comme, par exemple, la réalisation d'un mini sondage d'opinion auprès d'un échantillonnage de la population anglophone, ou encore, une interview.

Une fois les trois phases de réception calées, nous passons à la conception des deux phases de production. Pour la phase de production écrite, nous pourrions envisager comme tâche la rédaction d'un fax (grève des postes oblige !) par notre fan du Pink Floyd au copain à Paris qui va l'héberger, l'informant de son arrivée et les conditions du voyage. Contrairement au scénario d'évaluation, le scénario pédagogique présente beaucoup de souplesse par rapport aux critères définitoires de la phase de présentation et négociation orales. Celle-ci peut s'organiser autour d'un débat portant soit sur la problématique 
générale (la grève des transports) soit sur une problématique individuelle de mise en situation (existe-t-il d'autres façons de résoudre la problématique individuelle? Les autres entrevoient-ils d'autres possibilités? Quelqu'un aurait-il une voiture pour d'autres personnes intéressées par un voyage à Paris? Ou ailleurs ?...). Le profil de ce scénario pédagogique peut être résumé sous forme de schéma (cf. figure 2).

\section{Mise en situation}

"Vous êtes étudiant et fan du Pink Floyd. Votre grand-mère vous a offert, pour votre anniversaire, des billets pour un concert exceptionnel à Paris. Malheureusement, une grève générale rend difficile le voyage d'Aix à Paris. Vous devez essayer de trouver d'autres modes de transport pour vous rendre à Paris avec vos amis. »

Figure 2. Scénario pédagogique FLE, thématique générale. Exemple de support et de déroulement pour degrés DCL 2, $3 \& 4$

Pink Floyd - I Wanna Getchya

\begin{tabular}{|c|c|c|c|}
\hline Phase & Compétence & Support & Taches \\
\hline 1 & $\begin{array}{l}\text { compréhension } \\
\text { de l'écrit }\end{array}$ & $\begin{array}{l}\text { - article(s) de } \\
\text { la presse ecrite }\end{array}$ & $\begin{array}{l}\text { - recuell des informations } \\
\text { concernant l'état de paralysie } \\
\text { dans le système de } \\
\text { transports publics }\end{array}$ \\
\hline 2 & $\begin{array}{l}\text { compréhension } \\
\text { de l'oral }\end{array}$ & $\begin{array}{l}\text { extrait des } \\
\text { informations } \\
\text { télévisées ou } \\
\text { radio }\end{array}$ & $\begin{array}{l}\text { - recueil des informations } \\
\text { relatives à la mise en place } \\
\text { des transports de } \\
\text { substitutions }\end{array}$ \\
\hline 3 & $\begin{array}{l}\text { interaction } \\
\text { orale }\end{array}$ & $\begin{array}{l}\text { - Interaction } \\
\text { entre paires ou } \\
\text { conversation } \\
\text { «téléphonique» } \\
\text { en laboratoire }\end{array}$ & $\begin{array}{l}\text { - demande des précisions } \\
\text { concernant les transports de } \\
\text { substitution (heure et lieu de } \\
\text { départ, coôt, éventualité } \\
\text { d'organiser le retour...) }\end{array}$ \\
\hline 4 & $\begin{array}{l}\text { production } \\
\text { écrite }\end{array}$ & - aucun & $\begin{array}{l}\text { - envol de fax à la personne } \\
\text { quil va l'héberger l'informant } \\
\text { de son arrivée et les } \\
\text { conditions de son voyage }\end{array}$ \\
\hline 5 & $\begin{array}{l}\text { présentation et } \\
\text { négoclation } \\
\text { orales }\end{array}$ & - aucun & $\begin{array}{l}\text { - débat autour de la } \\
\text { problématique générale (la } \\
\text { grève des transports) } \\
\text { ou } \\
\text { façons de résoudre la } \\
\text { problématique individuelle }\end{array}$ \\
\hline
\end{tabular}

\subsection{Fabrication des grilles de guidage}

Les activités de réception sont centrées sur le recueil d'informations ciblées en fonction d'une problématique précise, à l'inverse du recueil d'informations à objectif général, non ciblé. Cette distinction est importante dans le domaine de la didactique : elle correspond à la volonté de traduire en activité pédagogique une activité réelle qui consiste à lire pour trier, classer et hiérarchiser les informations surabondantes auxquelles nous sommes tous confrontées aujourd'hui.

Un scénario se présente sous forme de petit fascicule, format A4. La première page est automatiquement la mise en situation, suivie des documents qui constituent la phase de la compréhension de l'écrit. Cette phase, ainsi que les deux autres phases de réception, est accompagnée d'une grille de guidage destinée à aider l'apprenant à mieux cibler son 
objectif et à mener à bien cette tâche complexe (tri, classement et hiérarchisation des informations).

Nous pourrions imaginer la grille de guidage se rapportant aux documents de la phase de compréhension de l'écrit, par exemple, de notre scénario hypothétique comme dans le tableau 1.

Tableau 1. Information Grid - Phase 1

This grid is to help you select the information you need to reach your decision

\begin{tabular}{|l|}
\hline - Types of transport concerned: \\
\hline - Regions affected: \\
\hline - Towns affected \\
\hline - Possible duration of strike: \\
\hline - Possibility of negotiations: \\
\hline - Indications concerning possible end of strike: \\
\hline
\end{tabular}

Nous remarquerons d'une part, le formatage de la grille et, d'autre part, au niveau du langage, l'extrême économie de mots: une formulation dépouillée, une syntaxe peu développée et l'absence du recours à la forme interrogative. Cela correspond à une volonté de se conformer aux habitudes de la vie courante où, en pareille situation, nous recourons davantage aux formulations « cryptiques » (ex. « send fax tomorrow » ou " call John before 12 » ou "check flight schedule») qu'aux formulations complexes et complètes. Il en sera de même pour les grilles de guidage qui accompagnent la phase de la compréhension de l'oral et la phase d'interaction. Les phases de production (écrite et orale) d'un scénario pédagogique ne nécessitent pas de grilles de guidage.

\section{Organisation du scénario et son exploitation en unités d'enseignement}

Pour ce qui concerne les détails pratiques d'organisation du cours, le scénario est un outil très modulable. Il peut être fractionné en plusieurs configurations selon la durée du cours et le niveau des étudiants :

- 6 unités : la mise en situation et chaque phase constitue une unité et couvre une séance

- 5 unités : chaque phase est une unité et couvre une séance

- 4 unités : la phase de compréhension de l'écrit constitue un devoir à faire à la maison

- 3 unités : la phase de production écrite se fait également à la maison

- 2 unités : les phases de réception audio et interactionnelle se font dans une première séance, l'écrit à la maison, et le débat pendant une deuxième séance 


\section{Pour qui ?}

\section{Quelques adaptations en fonction de la langue enseignée et/ou du public ciblé}

Ayant fait une démonstration, que nous espérons convaincante, de l'extrême faisabilité de l'exploitation du scénario comme outil pédagogique, il serait intéressant, à partir des éléments qui précédent, de se pencher sur les possibilités d'adaptation que cet outil offre dans les domaines plus pointus de l'enseignement des langues. Je vous propose, par conséquent, de se pencher sur trois secteurs qui suscitent un intérêt considérable aujourd'hui : l'enseignement des langues dites rares (modimes), l'enseignement des langues de spécialité et l'enseignement en auto-formation guidée.

\subsection{Le scénario dans l'enseignement des langues dites rares}

Par le terme «langues rares", nous entendons toutes les langues à l'exception des cinq langues européennes majoritairement enseignées. Plus précisément, il s'agit d'examiner le potentiel d'exploitation qu'offre le scénario pédagogique dans le cadre de l'enseignement des langues dites « difficiles » en raison de leur alphabet non romain.

Une considération importante est le fait qu'il existe peu de public de niveau avancé dans l'enseignement de ces langues. Ainsi, se greffe sur la question de la faisabilité, celle du calibrage de difficulté du scénario.

En partant de l'hypothèse que plus les informations sont de nature factuelle, plus la tâche est calibrée vers le bas (à l'inverse, plus les informations sont de nature conceptuelle, plus la tâche est calibrée vers le haut), nous avons imaginé un scénario pour un public de japonais faux débutants (environ 2 à 3 ans d'enseignement en milieu institutionnel). Le thème choisi est encore celui du voyage et la problématique est définie comme suit (voir figure 3).

Voyage to the land of the Rising Sun

Mise en situation

À Tokyo en voyage d'affaires, vous recevez une lettre des dirigeants d'une société située à Nagasaki qui souhaitent vous rencontrer pour mieux connaître les activités de votre société. Vous avez deux possibilités de transport : le train et l'avion. De plus, vous devez réserver une chambre d'hôtel à Nagasaki. Une fois que vous avez arrêté les modalités de votre voyage, vous devez envoyer un fax à vos hôtes à Nagasaki, les informant de l'heure de votre arrivée et de l'hôtel que vous auriez choisi. 
Figure 3. Scénario pédagogique dans le domaine des langues rares (modimes) : le japonais Exemple de support et de déroulement pour Degrés DCL 1 \& 2

\begin{tabular}{|c|c|c|c|}
\hline Phase & Compétence & Support & Tâche \\
\hline 1 & $\begin{array}{l}\text { compréhensio } \\
\text { n de l'écrit }\end{array}$ & $\begin{array}{l}\text { - horaires } \\
\text { d'avions } \\
\text { Tokyo- } \\
\text { Nagasaki } \\
\text { - liste d'hótels } \\
\text { et plan de } \\
\text { Nagasaki }\end{array}$ & $\begin{array}{l}\text { vérifler les horaires des } \\
\text { vols et choisir ceux qui } \\
\text { vous conviennent le } \\
\text { mieux } \\
\text { - choisir un hôtel près du } \\
\text { lleu du rendez-vous }\end{array}$ \\
\hline 2 & $\begin{array}{l}\text { Compréhensio } \\
\text { n de l'oral }\end{array}$ & $\begin{array}{l}\text { un } \\
\text { enregistremen } \\
\text { t quil récite les } \\
\text { heures de } \\
\text { départ des } \\
\text { trains pour } \\
\text { Nagasakl }\end{array}$ & $\begin{array}{l}\text { - choisir les horaires de } \\
\text { train qui vous } \\
\text { conviennent le mieux } \\
\text { choisir entre le train et } \\
\text { l'avion }\end{array}$ \\
\hline 3 & $\begin{array}{l}\text { interaction } \\
\text { orale }\end{array}$ & $\begin{array}{l}\text { - } \text { interaction } \\
\text { entre paires } \\
\text { ou avec } \\
\text { enseignant } \\
\text { ou } \\
\text { - Interaction au } \\
\text { téléphone }\end{array}$ & $\begin{array}{l}\text { réserver votre billet } \\
\text { d'avion ou train } \\
\text { ou } \\
\text { téléphoner à l'hôtel pour } \\
\text { réserver votre chambre } \\
\text { en précisant la durée de } \\
\text { votre séjour }\end{array}$ \\
\hline 4 & $\begin{array}{l}\text { production } \\
\text { écrite }\end{array}$ & - aucun & $\begin{array}{l}\text { envoyer un fax à } \\
\text { Nagasaki informant vos } \\
\text { hótes de la date et de } \\
\text { l'heure de votre arrivée } \\
\text { ainsi que le nom de votre } \\
\text { hôtel }\end{array}$ \\
\hline 5 & $\begin{array}{l}\text { présentation } \\
\text { orale }\end{array}$ & - aucun & $\begin{array}{l}\text { - se présenter aux } \\
\text { personnes assistant à la } \\
\text { réunion }\end{array}$ \\
\hline
\end{tabular}

\subsection{Le scénario dans l'enseignement des langues de spécialité}

Une autre exploitation possible concerne l'adaptation du scénario aux enseignements dispensés dans le cadre du LANSAD ${ }^{3}$, notamment dans le vaste domaine des langues de spécialité. L'impact de l'approche communicative a fortement marqué ce secteur et nous assistons, depuis les années 80 , à un déplacement du centrage sur l'enseignant et sur la langue vers l'apprenant et ses besoins. Ainsi, la focalisation ne porte plus sur l'aspect lexical et/ou le déchiffrage des textes techniques, comme c'était souvent le cas auparavant, et aujourd'hui la notion de besoins se définit davantage par rapport à un contexte socioprofessionnel bien plus élargi. Se pose, ainsi, dans ce domaine si longtemps considéré comme «maigre» la question de la mise en œuvre d'une approche communicative articulée autour de la notion des tâches communicatives. Le scénario répond parfaitement à ce besoin.

Il existe un nombre quasiment inquantifiable de langues de spécialité. Nous avons choisi d'explorer le potentiel d'adaptation du scénario par rapport à celle de la pharmacie pour démontrer que même dans un domaine relativement moins exploré que, par exemple, le droit, les affaires, la banque, etc., il est tout à fait possible d'introduire une dynamique autre.

La thématique choisie est celle d'un « consommateur » qui cherche à se renseigner sur un nouveau médicament vendu sans ordonnance aux États-Unis, la mélatonine. En ciblant des étudiants se situant autour des degrés 2,3 \& 4 du DCL, la mise en situation pourrait définir la problématique du scénario comme dans la figure 4 . 


\section{Mise en situation}

Vous êtes étudiant en pharmacie et postulez à une bourse aux États-Unis. Pour ce faire, vous devez présenter une enquête sur un médicament nouveau sous forme d'étude de cas: un ingénieur travaillant pour une société internationale est appelé à voyager fréquemment pour le compte de celle-ci. Malheureusement, il souffre particulièrement des effets du décalage horaire. Il a entendu parler d'un nouveau médicament commercialisé librement aux États-Unis qui serait très efficace, non seulement contre les effets du décalage horaire, mais également contre la fatigue et le vieillissement en général. Il existe plusieurs marques. Votre ingénieur doit partir prochainement aux ÉtatsUnis et souhaite se procurer ce médicament lors de son voyage. Il hésite cependant parce qu'il craint que le mélatonin n'induise d'autres effets secondaires, notamment la somnolence. Vous devez vous documenter quant à l'efficacité de ce médicament et les contre-indications par rapport à ses appréhensions.

Figure 4. Scénario pédagogique dans le domaine des langues de spécialité (pharmacie). Exemple de support et de déroulement pour Degrés DCL 2, 3 \& 4

\begin{tabular}{|c|c|c|c|}
\hline Phase & Compétence & Support & Taches \\
\hline 1 & $\begin{array}{l}\text { compréhension } \\
\text { de l'écrit }\end{array}$ & $\begin{array}{l}\text { - } 2 \text { articles de la presse } \\
\text { non spécialiste qui } \\
\text { traitent le sujet sur le } \\
\text { plan général } \\
\text { - } 2 \text { notices techniques } \\
\text { détalliées ( } 2 \text { pages } \\
\text { chacune) des deux } \\
\text { marques les plus } \\
\text { vendues de mélatonin } \\
\text { aux États Unis }\end{array}$ & $\begin{array}{l}\text { - recuellilir des informations } \\
\text { générales relatives à ce } \\
\text { produit } \\
\text { - vérifier les conditions de } \\
\text { vente } \\
\text { - vérifier les indications, } \\
\text { contre indications, mises en } \\
\text { garde et précautions } \\
\text { d'emplol }\end{array}$ \\
\hline 2 & $\begin{array}{l}\text { compréhension } \\
\text { de l'oral }\end{array}$ & $\begin{array}{l}\text { - un document vidéo } \\
\text { (ou audio) }\end{array}$ & $\begin{array}{l}\text { recueililir l'avis donné par } \\
\text { deux spécialistes lors d'un } \\
\text { interview télévisé }\end{array}$ \\
\hline 3 & $\begin{array}{l}\text { nteraction } \\
\text { orale }\end{array}$ & $\begin{array}{l}\text { - Interview avec paires } \\
\text { ou sondage } \\
\text { téléphonique }\end{array}$ & $\begin{array}{l}\text { - recueillir l'avis d'un certain } \\
\text { nombre d'utilisateurs }\end{array}$ \\
\hline 4 & $\begin{array}{l}\text { production } \\
\text { écrite }\end{array}$ & - aucun & $\begin{array}{l}\text { - rédiger un rapport sur les } \\
\text { avantages et les } \\
\text { inconvénients du produit }\end{array}$ \\
\hline 5 & $\begin{array}{l}\text { présentation } \\
\text { orale }\end{array}$ & - aucun & $\begin{array}{l}\text { * présenter ou soutenir son } \\
\text { rapport } \\
\text { ou } \\
\text { présenter ses conclusions et } \\
\text { répondre aux questions de } \\
\text { l'auditoire }\end{array}$ \\
\hline
\end{tabular}

La pharmacie est l'un des domaines des langues de spécialité relativement « discrets » ne serait ce que par le fait qu'un médicament défraie moins souvent la chronique que d'autres domaines des langues de spécialité plus "médiatisés ", tel que la justice ou l'économie, qui offrent, par conséquent, un potentiel en documentation encore plus riche et propice à l'exploitation du scénario. Ainsi, des disciplines aussi diversifiées et hétéroclites que le droit, l'économie, la médecine, la géologie, l'informatique, etc., laissent entrevoir des perspectives très intéressantes à cet égard. 


\subsection{Le scénario et l'enseignement en autonomie guidée} l'enseignement des langues d'aujourd'hui et de demain. Cependant, il s'agit d'un type d'enseignement qui obéit à de nouvelles contraintes et exige, par conséquent, de nouvelles approches, méthodologies et applications didactiques. L'utilisation du scénario en tant qu'outil pédagogique peut apporter une réponse à ces nouveaux besoins.

L'enseignement-apprentissage en autonomie guidée est un concept qui permet une très grande flexibilité au niveau des configurations pédagogiques et organisationnelles possibles. Dans le cadre de notre réflexion ici, nous limiterons notre étude du scénario appliqué à l'autonomie guidée à deux configurations qui ont déjà été expérimentées dans le cadre des enseignements de langue dispensés au Centre de Langues Vivantes pour des étudiants inscrits à la Faculté de Droit de l'Université Pierre Mendes France, Grenoble II :

- l'autoformation guidée en « semaine alternée »

- l'autoformation «semi-guidée » autour d'un projet.

\subsubsection{L'autoformation guidée en « semaine alternée »}

Née à l'origine de la simple nécessité de scinder des "grands» groupes en deux (30 en deux groupes de 15), cette forme d'apprentissage-enseignement est conçue sur le principe d'un présentiel fréquent et rapproché, c'est à dire, une semaine sur deux. Il s'agit d'une formule d'auto-apprentissage qui convient plus particulièrement aux apprenants ayant encore besoin d'encadrement et de guidage, soit en raison de leur niveau dans la langue soit en raison de leur maturité.

La formule de «semaine alternée» organise les différentes tâches communicatives qui composent le scénario autour d'une unité de deux séances: les tâches à accomplir pendant la première semaine ne nécessitent pas d'interaction, (recueil d'informations), et l'apprenant les accomplit à la médiathèque (presse, audio-vidéo). La deuxième séance est une séance "contact » centrée sur des activités d'interaction: l'apprenant recueille des informations spécifiques à la phase interactionnelle et ensuite, à l'aide des éléments recueillis, participe à un débat ou un jeu de rôle. Il réalise la partie écrite «à la maison » et la soumet à l'enseignant pour correction par la suite.

\subsubsection{L'autoformation « semi-guidée » autour d'un projet}

La formule d'autoformation « semi-guidée » est axée sur le principe d'un présentiel réduit et des rencontres enseignant-apprenant moins rapprochées (certaines formules ne prévoient que trois rencontres par an), et se destine plus particulièrement aux apprenants ayant déjà acquis à la fois une certaine maîtrise de la langue et une certaine maturité dans la gestion du temps de travail. Sa très grande flexibilité la rend également idéale pour l'établissement de «contrats pédagogiques» établis spécifiquement à l'égard de tel ou tel étudiant.

En fonction de la documentation disponible au centre de ressources dont dépend l'apprenant et/ou l'enseignant, un «projet de recherche» est communément établi entre l'enseignant et l'apprenant. A des fins d'illustration, nous supposerons que la thématique arrêtée concerne l'affaire O.J. Simpson et l'angle d'approche défini comme "L'argent 
comme facteur déterminant de l'acquittement d'O. J. Simpson ". L'élaboration du projet, conçue autour des cinq phases du scénario, s'organise en quatre étapes (voir figure 5).

Figure 5. Scénario pédagogique dans le domaine de l'autonomie semi-guidée (présentiel réduit). Exemple de support et de déroulement pour degrés DCL 2, 3 \& 4

OJ Simpson - How much for an acquittal?

\begin{tabular}{|c|c|c|c|}
\hline \multicolumn{4}{|c|}{$\begin{array}{l}\text { Premier entretien avec l'enseignant : définition de la problématique et de la } \\
\text { méthodologie de travali. Indications sur la documentation existante }\end{array}$} \\
\hline Phase & Compétence & Support & Tâches \\
\hline $1 \& 2$ & \begin{tabular}{|l|} 
Recuell \\
d'informations \\
écrites et orales
\end{tabular} & $\begin{array}{l}\text { articles de la presse } \\
\text { non-spécialiste } \\
\text { entretiens télévisés } \\
\text { vidéo cassette d'OJ } \\
\text { Simpson lui-même }\end{array}$ & $\begin{array}{l}\text { recueillir des informations } \\
\text { relatives à la problématique } \\
\text { à étudier }\end{array}$ \\
\hline \multicolumn{4}{|c|}{$\begin{array}{c}\boldsymbol{Z} \text { entretien avec l'enseignant : bilan de la } 1^{*} \text { étape et définition des orlentations et } \\
\text { des modalités de la } 2 \text { étape }\end{array}$} \\
\hline 3 & interaction orale & sondage et interviews & $\begin{array}{l}\text { recueillir l'avis d'un certain } \\
\text { nombre d'anglophones et/ou } \\
\text { personnes ayant une } \\
\text { maitrise de la langue cible }\end{array}$ \\
\hline \multicolumn{4}{|c|}{$\begin{array}{c}\hat{F}^{*} \text { entretien (et le dernier avant la soutenance) avec l'enseignant: bilan de la } \boldsymbol{Z}^{\mathbf{x}} \text { étape } \\
\text { et définition des critères requis pour l'évaluation du rapport. Attentes par rapport à la } \\
\text { soutenance }\end{array}$} \\
\hline 4 & production écrite & aucun & $\begin{array}{l}\text { rédiger un rapport d'environ } \\
2000 \text { à } 2500 \text { mots sur la } \\
\text { problèmatique définie }\end{array}$ \\
\hline $\begin{array}{c}5 \\
\text { (et } 4 \mathrm{e} \\
\text { rencontre) }\end{array}$ & présentation orale & aucun & $\begin{array}{l}\text { faire une brève présentation } \\
\text { de ses travaux et soutenir } \\
\text { son rapport }\end{array}$ \\
\hline
\end{tabular}

Les rencontres enseignant-apprenant peuvent être espacées pour correspondre au démarrage de chaque nouvelle phase du scénario, ce qui porte le nombre de rencontres à un minimum de quatre, y compris la séance de soutenance.

\section{Conclusion}

L'adaptation du scénario comme outil pédagogique est fondée sur le paradoxe de la contrainte et de la liberté. D'un côté, il est, certes, indéniable que l'utilisation de ce type d'outil pédagogique est conditionnée par l'existence d'une banque de données bien garnie, ce qui suppose un effort collectif, dans le temps et sur le budget. Mais cette contrainte est bien la seule et la sensibilisation de tous les partenaires, les institutions, les enseignants et les apprenants à la nécessité d'explorer des voies nouvelles, conjuguée à la diffusion croissante de documents de toute sorte, sont autant d'éléments qui permettent d'afficher un certain optimisme - aussi prudent soit-il - à l'égard de l'évolution à espérer dans ce domaine.

Il ne reste alors que la liberté. La liberté d'innover, d'expérimenter, de faire et se faire plaisir en créant une nouvelle dynamique pédagogique. 


\section{BIBLIOGRAPHIE}

Isani, S. \& M. Herino. 1994. « La notion de scénario comme outil d'évaluation de la compétence en langue ». ASp 3, 101-121.

Prodromou, L. 1995. « The backwash effect: From test to teaching ». ELT Journal 49/1, 13-25.

\section{NOTES}

1. Voir Isani \& Herino (1994).

2. Nous rappelons ici que le scénario en tant qu'outil pédagogique et outil d'évaluation est exploité depuis un certain temps déjà, notamment au Canada dans le contexte du FLE.

3. LANSAD : LANgues pour Spécialistes d'Autres Disciplines, terme plus clair et valorisant que «non-spécialiste» (acronyme forgé par M. Perrin au colloque d'Aix sur «L'enseignement des langues aux non spécialistes » en février 1993).

\section{RÉSUMÉS}

Une des options fortes du Diplôme de compétence en langue (DCL) fut de relever le défi d'évaluer la compétence en langue par le biais du scénario, un outil d'évaluation relativement innovant. Nous examinons ici le potentiel que peut offrir l'exploitation du scénario dans le contexte pédagogique de l'enseignement-apprentissage des langues étrangères, notamment dans les domaines des langues rares, les langues de spécialité et la formation en autonomie guidée.

One of the major options of the French Diplôme de compétence en langue (DCL) was to evaluate communicative competence in foreign languages through a relatively new and unexplored evaluation tool, the scenario. The object of this article is to explore the potential offered by this tool in the field of foreign language learning and teaching and its applications in specific fields such as minor languages, ESP and guided self-learning.

\section{INDEX}

Mots-clés : anglais de spécialité, formation en autonomie, langue rare, pédagogie, scénario

Keywords : ESP, minor language, scenario for teaching purposes, self-learning 


\section{AUTEUR}

\section{SHAEDA ISANI}

Shaeda Isani enseigne à l'Université Stendhal, Grenoble 3. shaeda.isani@u-grenoble3.fr 